\title{
Triple combination antibiotic therapy for carbapenemase-producing Klebsiella pneumoniae: a systematic review
}

\author{
David M. Jacobs ${ }^{1 *}$, M. Courtney Safir ${ }^{1}$, Dennis Huang ${ }^{1}$, Faisal Minhaj ${ }^{1}$, Adam Parker ${ }^{1}$ and Gauri G. Rao ${ }^{2 *}$
}

\begin{abstract}
Background: The spread of carbapenemase-producing K. pneumoniae (CPKP) has become a significant problem worldwide. Combination therapy for CPKP is encouraging, but polymyxin resistance to many antibiotics is hampering effective treatment. Combination therapy with three or more antibiotics is being increasingly reported, therefore we performed a systematic review of triple combination cases in an effort to evaluate their clinical effectiveness for CPKP infections.
\end{abstract}

\begin{abstract}
Methods: The PubMed database was searched to identify all published clinical outcomes of CPKP infections treated with triple combination therapy. Articles were stratified into two tiers depending on the level of clinical detail provided. A tier 1 study included: antibiotic regimen, regimen-specific outcome, patient status at onset of infection, and source of infection. Articles not reaching these criteria were considered tier 2.
\end{abstract}

Results: Thirty-three studies were eligible, 23 tier 1 and ten tier 2. Among tier 1 studies, 53 cases were included in this analysis. The most common infection was pneumonia (31\%) followed by primary or catheter-related bacteremia (21\%) and urinary tract infection (17\%). Different combinations of antibiotic classes were utilized in triple combinations, the most common being a polymyxin (colistin or polymyxin B, 86.8\%), tigecycline $(73.6 \%)$, aminoglycoside (43.4\%), or carbapenem (43.4\%). Clinical and microbiological failure occurred in 14/39 patients (35.9\%) and 22/42 patients (52.4\%), respectively. Overall mortality for patients treated with triple combination therapy was 35.8\% (19/53 patients).

Conclusions: Triple combination therapy is being considered as a treatment option for CPKP. Polymyxin-based therapy is the backbone antibiotic in these regimens, but its effectiveness needs establishing in prospective clinical trials.

Keywords: Triple combination treatment, KPC, Antibiotic resistance, Carbapenemase-producing K. pneumoniae

\section{Background}

The increasing global prevalence of carbapenem-resistant Enterobacteriaceae (CRE) combined with the decline in effective therapies is a public healthcare crisis. Infections caused by these multi-drug resistant (MDR) Gram-negative bacteria are associated with high mortality rates, often estimated at $40 \%$ or higher [1-7]. Since its discovery, CRE has spread to every continent and is now endemic in certain areas [1, 8-10]. A common

\footnotetext{
*Correspondence: dmjacobs@buffalo.edu; gaurirao@live.unc.edu ${ }^{1}$ Department of Pharmacy Practice, University at Buffalo School of Pharmacy and Pharmaceutical Sciences, Buffalo, NY, USA

${ }^{2}$ Division of Pharmaceutics and Experimental Therapeutics, Eshelman School of Pharmacy, University of North Carolina, Chapel Hill, NC, USA
}

mechanism of resistance in CRE is the production of carbapenemase enzymes, which confer resistance to many of the currently available antibiotics and limit treatment options [3, 8, 11, 12]. Among Enterobacteriaceae, carbapenemase production is common in Klebsiella pneumoniae $[3,8,9]$. The concurrent administration of multiple antibiotic agents can increase pharmacodynamic killing activity and potentially suppress or delay the emergence of resistance by broadening the spectrum of activity and exploiting different mechanisms of action [2, 4, 11, 13]. In the absence of evidence-based treatment guidelines, clinicians are increasingly resorting to using combination therapy for difficult-to-treat infections on the basis of some weak but promising published data $[2,4,6,11]$. 
There is currently no consensus on the most appropriate treatment for infections caused by carbapenemaseproducing K. pneumoniae (CPKP) [3, 6]. A recent review showed a mortality benefit with the use of combination therapy for CPKP, which is encouraging [11]. However, the evidence is conflicting, as a different comprehensive review found similar mortality in patients whether treated with monotherapy or combination therapy [13]. As the minimum inhibitory concentrations (MICs) to many antibiotics continue to creep upwards and with recent reports of polymyxin resistance, treating infections caused by carbapenemase producers is increasingly challenging [14, 15] and may necessitate combination therapy of three or more antibiotics in the near future. In vitro studies have shown promising results for the treatment of highly resistant $\mathrm{KPC}$-producing organisms utilizing triple drug combinations [16, 17], but clinical studies on the treatment of CPKP infections utilizing three or more antibiotics are scarce and mainly limited to case reports or small numbers of cases within larger studies. Therefore, we performed a systematic review of individual cases in an effort to evaluate the effectiveness of combination treatment regimens of three or more antibiotics (triple combination) on clinical outcomes for CPKP infections.

\section{Methods}

\section{Literature search}

A systematic review was conducted using the PubMed database from inception to March [1], 2016 using the following search terms: "carbapenemase-producing Klebsiella pneumoniae", "carbapenem-resistant Klebsiella pneumoniae", and "KPC". Only articles published in English or translated to English were evaluated. The bibliographies of all eligible studies were reviewed in an effort to identify other eligible studies.

\section{Study selection}

Articles were eligible if they included any patients with infections due to carbapenemase-producing K. pneumoniae treated with triple combination antimicrobial therapy for a minimum of $48 \mathrm{~h}$. When an article included patients treated with triple combination therapy along with mono or dual antimicrobial therapy, only the triple combination data were extracted. Articles were excluded from further review if they fulfilled any of the following criteria: (1) the study evaluated Enterobacteriaceae other than K. pneumoniae; (2) the study included data based on only in vitro or in vivo infection models; (3) the details regarding the treatment regimens were not specified or included in the article; and (4) if the study only reported on patients colonized with CPKP.
Following initial identification, all potential articles were reviewed to assess their eligibility based on treatment and microbiology-specific exclusion criteria. Articles were ineligible if they fulfilled any of the following criteria: (1) carbapenemase production was not confirmed; (2) data related to CPKP could not be separated from other carbapenemase-producing Enterobacteriaceae; and (3) details regarding treatment with monotherapy or dual combination therapy specifically for the CPKP infection were included. One article was excluded as an updated version of the same study population was published soon afterwards. The authors were contacted in cases where details regarding the concurrent administration of antibiotics as a part of the triple combination were not clearly stated or could not be verified.

\section{Data extraction and definitions}

The data extracted from each article included the main characteristics of the: (1) study (first author name, publication year, country of origin, study period and design); (2) case (including age, sex, type of infection, and APACHE II score); and (3) antibiotic treatment. Antimicrobial agents were categorized based on antibiotic class: polymyxins, carbapenems, tigecycline, aminoglycosides, beta-lactam plus beta-lactamase inhibitors, fosfomycin, trimethoprim-sulfamethoxazole, and fluoroquinolones. Clinical outcomes including clinical failure, microbiological failure and mortality were also recorded. Clinical and microbiological failure were defined according to the definitions used by the investigators of the included study. Any indeterminate outcome as listed by the study authors was categorized as a failure. Overall mortality as reported by the study authors was also recorded.

\section{Results}

The results of the literature search and the process of selection of included publications are shown in Fig. 1. A total of 736 articles were retrieved from PubMed using the search terms listed, and 668 articles were excluded because articles were not available in English $(\mathrm{n}=33)$, Enterobacteriaceae other than Klebsiella pneumoniae were studied $(\mathrm{n}=20)$, in vitro $(\mathrm{n}=116)$ and in vivo $(n=13)$ models of infection were used, details regarding the clinical therapy were missing or not provided $(\mathrm{n}=476)$, and publications were not related to patients colonized with bacteria of interest $(n=10)$. Based on the initial search criteria, nine articles were retrieved based on our review of the bibliographies of articles included. Based on the second set of exclusion criteria, 44 additional publications were excluded because we could not: (1) confirm carbapenemase production $(\mathrm{n}=3)$, (2) separate CPKP data from other CRE infections $(n=2),(3)$ separate colonization data from infection data $(n=1)$, 

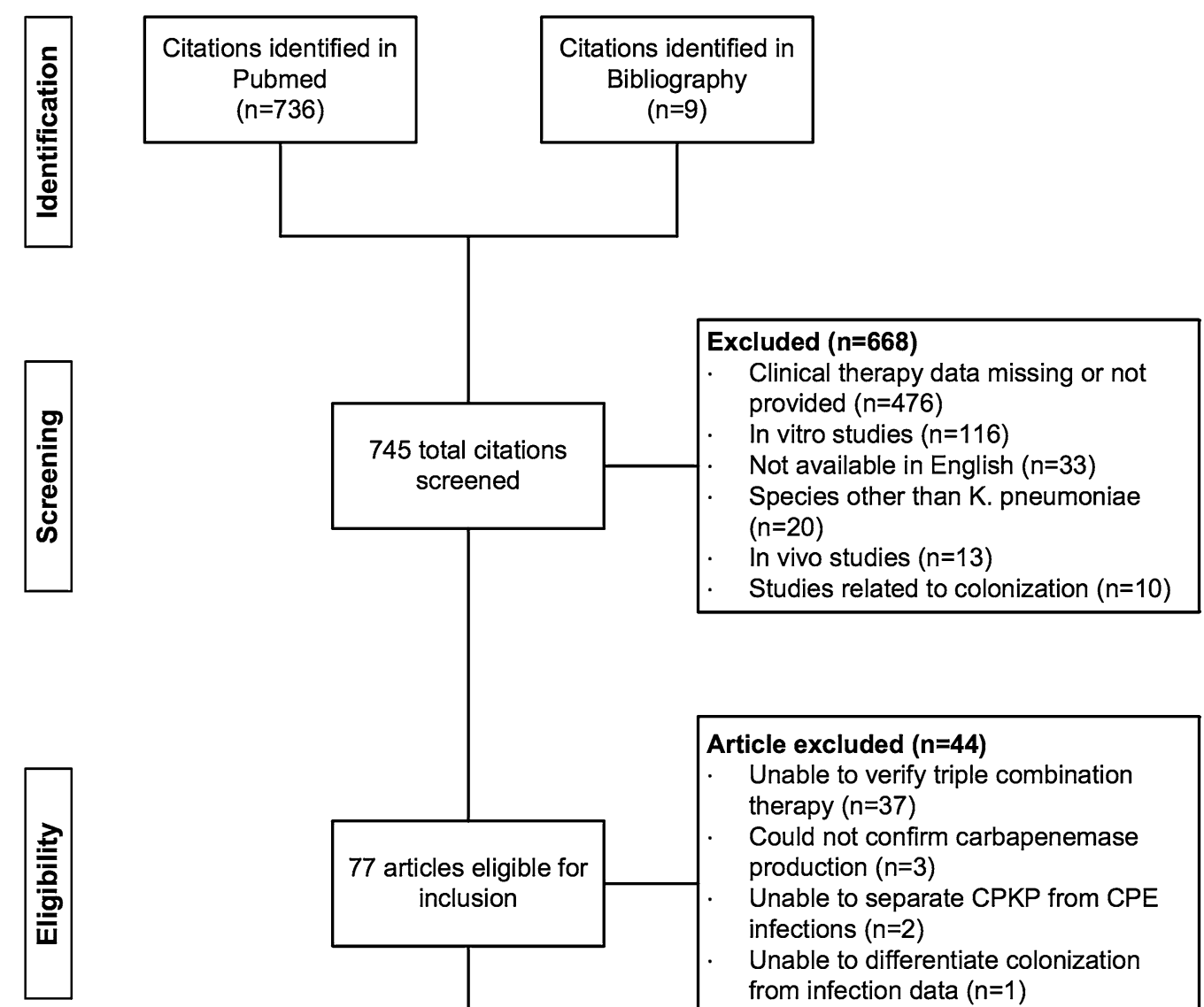

Article excluded $(n=44)$

Unable to verify triple combination therapy $(n=37)$

Could not confirm carbapenemase production $(n=3)$

Unable to separate CPKP from CPE infections $(n=2)$

Unable to differentiate colonization

from infection data $(n=1)$

Updated version available $(n=1)$

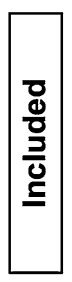

Fig. 1 Flow diagram of the search strategy and articles selected for review. CPKP, carbapenemase-producing K. pneumoniae; CPE, carbapenemaseproducing Enterobacteriaceae

and (4) verify concurrent administration of three agents ( $\mathrm{n}=37$ ).

The remaining 33 studies satisfied the minimum treatment duration requirement and were included in our final analysis. Thirteen studies were a combination of case reports or case series [18], fifteen were cohort studies [12, 31], and five were case-control studies [15, 45]. Study publication years ranged from 2009-2015, with the number of publications increasing in frequency over time, which coincided with an increasing trend in the percent of carbapenem-resistant $K$. pneumoniae isolates in the United States (Fig. 2). The country of origin for most of these studies was Greece $(n=9)$, the United States $(n=8)$, and Italy $(n=6)$ (Fig. 3$)$.

The articles included in this review were stratified into two tiers depending on the details of the reported treatment. A study classified as tier 1 included all of the following information: (1) antibiotic regimen, (2) regimen-specific outcome, (3) regimen-specific patient status at onset of infection, and (4) source of the infection. A study classified as tier 2 lacked information regarding one or more of these critical elements. 


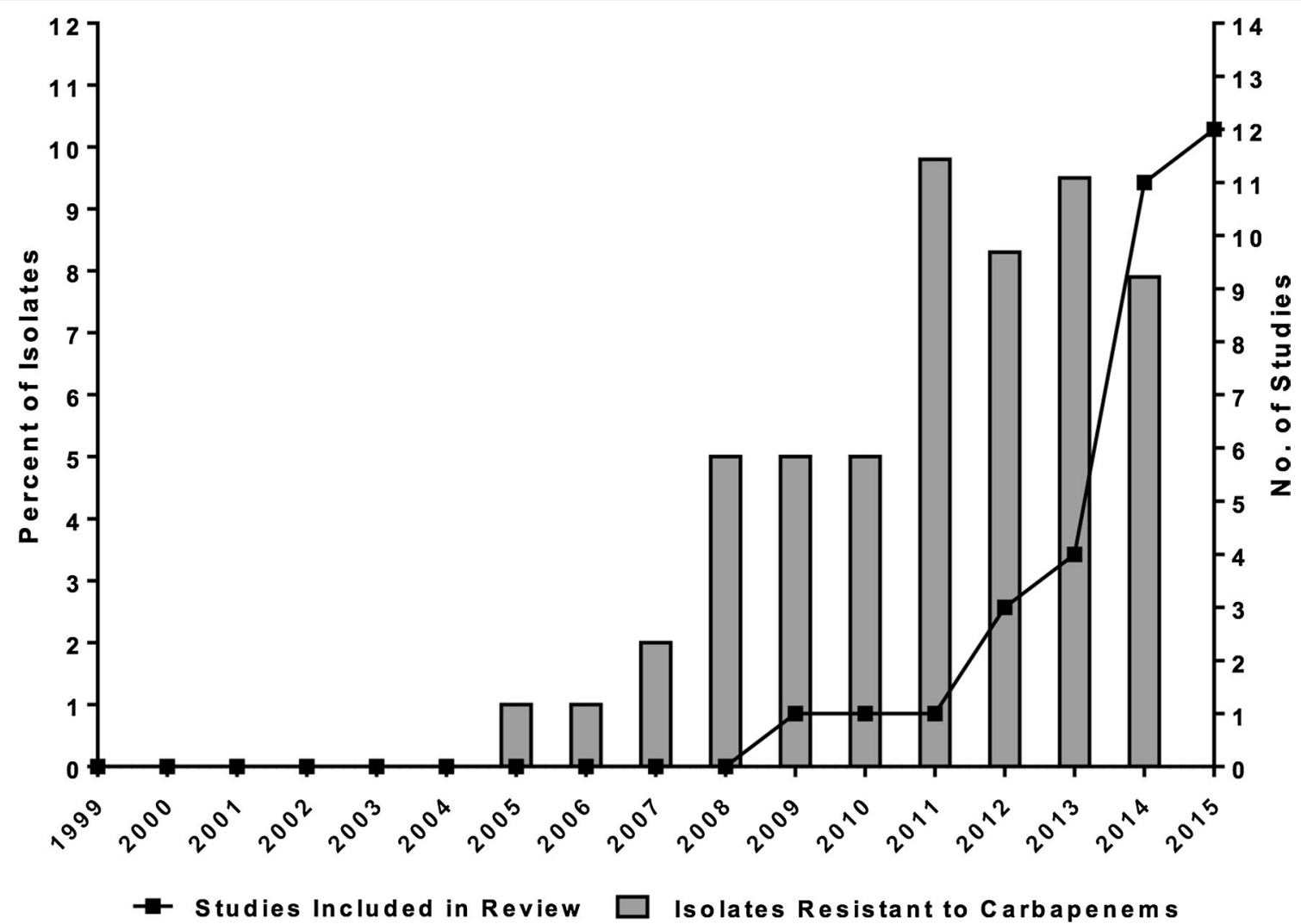

Fig. 2 Trends in the number of studies included in this review by publication year and the percentage of carbapenem-resistant K. pneumoniae isolates in the United States. Percent of resistant isolates is a composite of data from the Center for Disease Dynamics, Economics \& Policy and the Centers for Disease Control and Prevention

\section{Tier 1 results}

\section{Patient characteristics}

Twenty-three studies were classified as tier $1[18-33,37-$ $41,43,47]$ and comprised 53 patients (Table 1 ). The characteristics of these patients are presented in Table 2 . The mean age was $54 \pm 16$ years and $67 \%$ were males. Seventeen patients (63\%) had an APACHE II score $\geq 15$ and the median SOFA score was 10 (range 3-15). At time of onset of infection, six (25\%) patients were septic and 12 (50\%) patients had severe sepsis or septic shock.

\section{Clinical outcomes and antibiotic utilization}

Outcomes and antibiotic utilization stratified by publication for all patients in tier 1 are reported in Table 1 . Of the 53 patients included in tier 1 , clinical failure data were available for 39 patients, and 14 of these (35.9\%) failed on triple combination therapy. Microbiologic failure data were available for 43 patients, and 22 of these (52.4\%) failed on triple combination therapy. Overall, crude mortality among these patients was $35.8 \%$ (19/53). Polymyxins were the most frequently ( $47 / 53$ patients, $88.6 \%$ ) administered class of antibiotics among the different combination therapies followed by tigecycline (39/53 patients, $73.6 \%$ ), aminoglycosides (26/53 patients, $49.1 \%$ ), and carbapenems (23/43 patients, $43.4 \%)$. Rifampin and trimethoprim-sulfamethoxazole were used in one case each (1.9\%), with dual carbapenem therapy being utilized in three patients (5.7\%). Triple combination therapy was initiated empirically in six patients (11.3\%) and was provided as definitive treatment in 21 patients (39.6\%). Treatment sequence was not provided in 26 patients (49\%).

\section{Antimicrobial regimens by infection source}

Patient-specific antimicrobial regimens and outcomes stratified by infection source are presented in Table 3. Pneumonia was the most commonly reported (31\%) infection followed by primary or catheter-related bacteremia (21\%) and urinary tract infections (17\%). For pneumonia, patients were most commonly treated with a polymyxin (16/17 patients, 94\%) followed by tigecycline (13/17 patients, $76 \%)$. Overall, four patients with pneumonia died (24\%) either on or following treatment with combination antibiotic therapy. For the treatment 


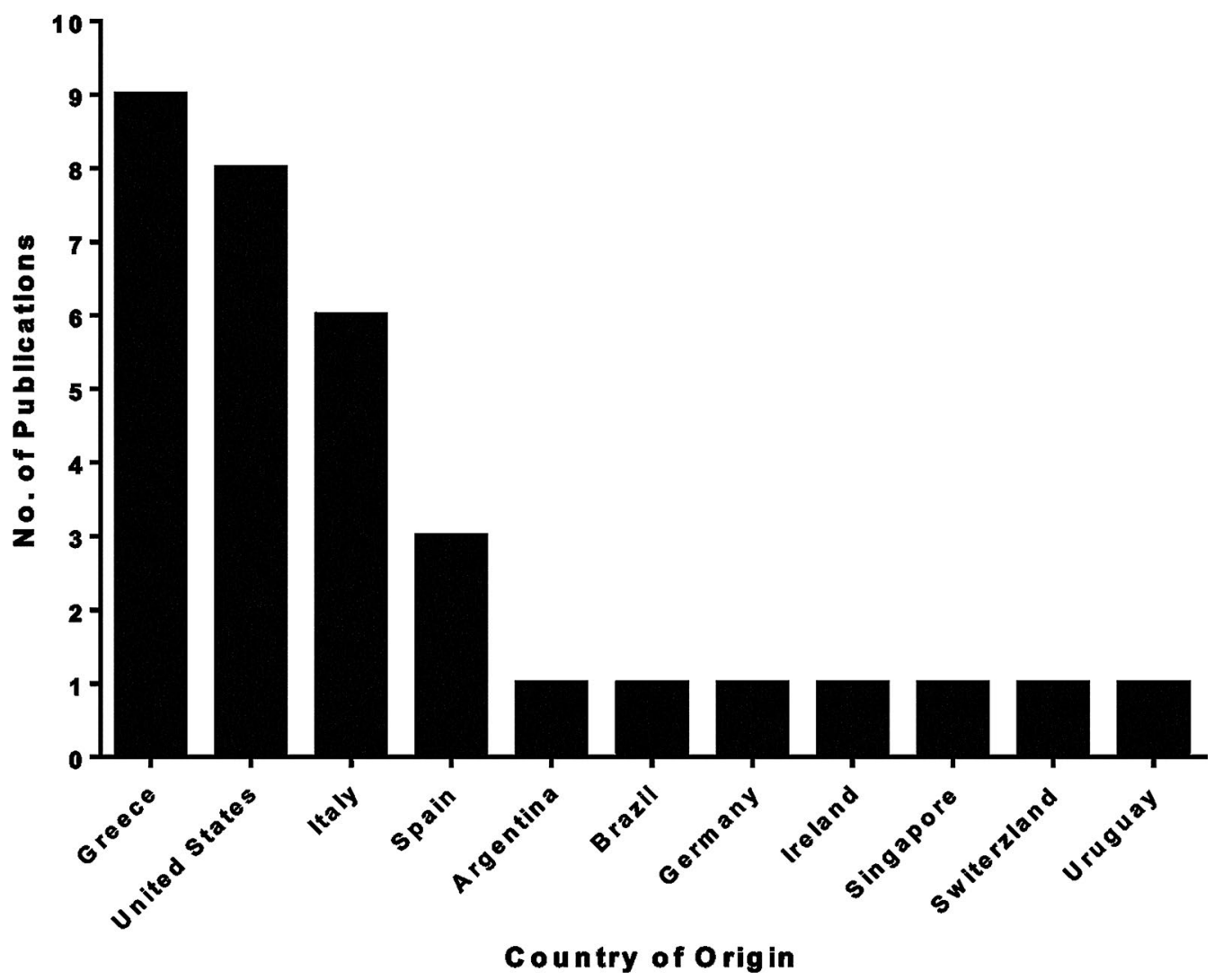

Fig. 3 Studies included in the review by country of origin $(n=33)$

of primary or catheter-related bacteremia, nine patients (9/11 patients, $82 \%)$ were treated with a polymyxin followed by meropenem (7/11 patients, 64\%). The crude mortality was $55 \%$ (6/11 patients) for patients with bacteremia. For the treatment of a urinary tract infection, polymyxin was commonly utilized (7/9 patients, $78 \%$ ) followed by meropenem (5/9 patients, $56 \%)$. Two patients (22\%) treated with combination therapy for a urinary tract infection died.

\section{Carbapenem use}

Carbapenem use and mortality stratified by meropenem MIC among tier 1 patients is presented in Fig. 4. The meropenem MIC data were not reported for 15 of the 53 patients. When a carbapenem was administered with a meropenem MIC $>8 \mathrm{mg} / \mathrm{L}, 10$ of $13(77 \%)$ patients were deemed a clinical success. Twenty-one patients were not administered a carbapenem where the meropenem MIC was $>8 \mathrm{mg} / \mathrm{mL}$. Among these patients, 12 of 21 (57\%) were deemed a clinical success. In patients where the meropenem $\mathrm{MIC}$ was $\leq 8 \mathrm{mg} / \mathrm{L}$, a carbapenem was administered in three cases, which resulted in clinical success in two of the cases.

\section{Tier 2 results}

The remaining ten studies were classified as tier $2[12,15$, 34-36, 42, 44-46, 48]. Data from these articles were difficult to gather since it was either missing or combined with patients on dual combination or monotherapy. These studies were categorized as tier 2 , since triple combination antibiotic therapy was utilized for the treatment of CPKP; however, the patient-specific and clinical details could not be ascertained.

\section{Discussion}

The widespread dissemination of Klebsiella pneumoniae carbapenemase producers has resulted in extensive spread of this resistant pathogen across the globe $[1,8]$. Our findings show a corresponding increase in the number of publications related to CPKP as resistance began to spread and worsen. This trend in publications was not only limited to Europe and the US, but also included South America and Southeast Asia. Furthermore, these strains are no longer confined to healthcare facilities but have spread to long-term care facilities and even to the community [49-52]. This rapidly growing problem also highlights the urgent need for new therapeutic strategies against resistant isolates. There is a growing interest and 


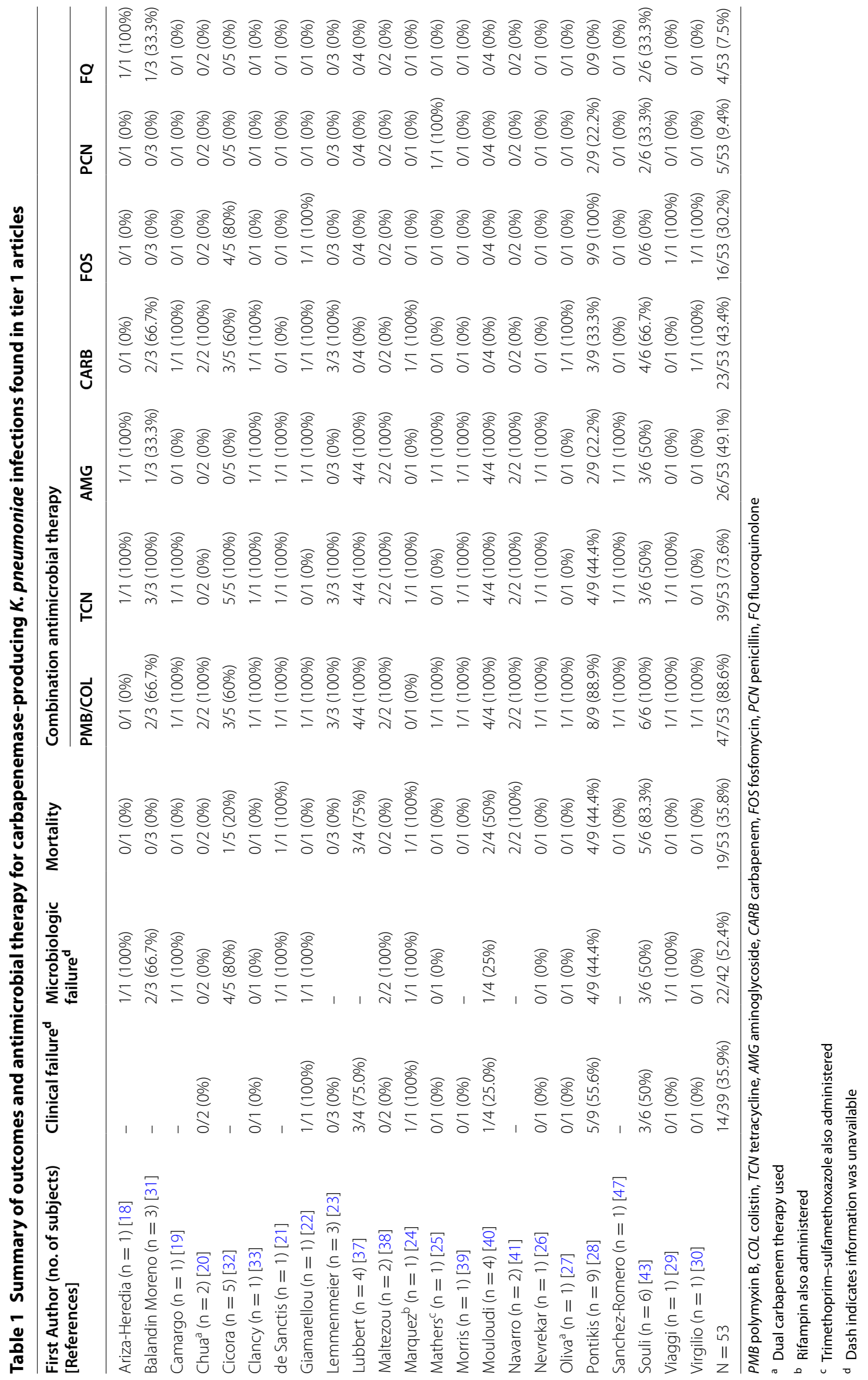


Table 2 Patient characteristics among tier 1 articles $(n=53)$

\begin{tabular}{ll}
\hline Characteristic & \\
\hline Age $(\mathrm{n}=53)^{\mathrm{a}}$ & $54 \pm 16^{\mathrm{b}}$ \\
Male Sex, $\mathrm{n}(\%)[\mathrm{n}=46]^{\mathrm{a}}$ & $31(67)$ \\
ICU admission, $\mathrm{n}(\%)[\mathrm{n}=38]$ & $33(87)$ \\
APACHE II Score $\geq 15, \mathrm{n}(\%)[\mathrm{n}=27]^{\mathrm{a}}$ & $17(63)$ \\
SOFA $(\mathrm{n}=18)^{\mathrm{a}}$ & $10(3-15)^{\mathrm{c}}$ \\
Sepsis syndrome at onset of infection, $\mathrm{n}(\%)(\mathrm{n}=24)$ & \\
Sepsis & $6(25)$ \\
Severe sepsis or septic shock & $12(50)$ \\
\hline
\end{tabular}

APACHE II Acute Physiology and Chronic Health Evaluation II, SOFA Sequential Organ Failure Assessment

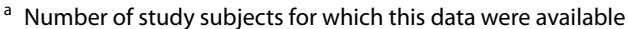

b Presented as mean \pm standard deviation

c Presented as median (range)

need to investigate combination therapies, so the aim of this review was to summarize available clinical data on the role of triple combination therapy in the treatment of infections due to carbapenemase producers.

The most appropriate antibiotic treatment regimens for the treatment of CPKP infections are not well defined. Our results show that triple combination therapy for the treatment of CPKP is being utilized with polymyxin as the common backbone antibiotic. The observed sustained pharmacodynamic in vitro activity of combination antibiotics that prevent the development of resistance has motivated clinicians to explore these promising combinations in their patients [16]. The current clinical treatment of infections due to these strains are largely based on clinical experience and observational studies. The increasing prevalence of carbapenemase-producing Enterobacteriaceae resistant to almost all available agents including polymyxins and tigecycline is highly concerning $[53,54]$. This has forced clinicians to reevaluate their treatment strategies against these highly resistant strains that cause infections associated with high mortality rates.

The Consortium on Resistance against Carbapenems in Klebsiella pneumoniae (CRACKLE) performed a prospective multicenter study that included 260 patients infected or colonized with carbapenem-resistant Klebsiella pneumoniae. The authors found that $39 \%$ of patients with bloodstream infection (BSI) or pneumonia died or were discharged to hospice [55]. Additionally, these patients were hospitalized for significantly longer periods, with an increased median total length of hospital stay of 5 and 10 days for BSI and pneumonia, respectively. Tzouvelekis et al. [11] reviewed the efficacy of combination therapy against infections due to carbapenemase-producing Enterobacteriaceae and, based on data from 889 patients, found that combination therapy was effective in 441 (48.6\%) patients compared to monotherapy in 346 (38.1\%) patients and $102(11.3 \%)$ patients who received inappropriate monotherapy with an antibiotic agent that had no in vitro activity against the infecting pathogen. Furthermore, monotherapy with carbapenem, tigecycline, or colistin were associated with unacceptably high mortality rates of $40.1,41.1$, and $42.8 \%$, respectively, similar to the high mortality of $46.1 \%$ observed in patients who received 'inappropriate' monotherapy [13]. Our findings are similar to these studies, with triple combination therapy considered clinically successful in 25 of 39 (64\%) patients. Also, the overall mortality among patients treated with triple combinations was $35.8 \%$, which is similar to findings from the CRACKLE study. In a study by Qureshi et al. [4], the most commonly used treatment combinations were colistin-polymyxin B or tigecycline combined with a carbapenem. Use of combination therapy resulted in lower mortality of $12.5 \%(1 / 8)$ compared to $66.7 \%(8 / 12)$ with polymyxin, carbapenem, or tigecycline alone [4]. The use of combinations for definitive therapy was associated with improved survival in bacteremia caused by KPC-producing $K$. pneumoniae. We also found that colistin-polymyxin B was the backbone antibiotic to many of these triple combination regimens along with tigecycline, highlighting the importance of polymyxins as a treatment option for these highly resistant organisms.

The increasing emergence of strains resistant to polymyxins and the development of polymyxin resistance on treatment are very concerning [56]. This is especially true as our findings show that polymyxins were commonly used as part of triple combination therapy for treating CРКP. Development of polymyxin resistance with an associated increase in mortality has been reported in multiple studies [14, 15, 57]. The observed association may be due to decreased polymyxin susceptibility or possibly differences in baseline patient characteristics, severity of infection, or lack of adequate empirical therapy. Regardless, the development of polymyxin resistance is alarming. Polymyxin resistance may be mediated by modification of outer membrane lipopolysaccharide or by increased production of capsular polysaccharide in K. pneumoniae [58]. Treatment of 12 patients infected with CRKP with polymyxin alone resulted in significant increases in polymyxin MICs for three of the 12 patients in a relatively short period of 5-21 days. This rapid change could be attributed to reinfection with a resistant strain under antibiotic selective pressure. This change in susceptibility was prevented in patients treated with a combination of polymyxin B and tigecycline [59].

A few emerging treatment options for CPKP infections appear promising. The most prominent new agent is ceftazidime-avibactam, a cephalosporin combined 
Table 3 Patient-specific summary of demographics, clinical outcomes, and combination treatment by infection source among tier 1 articles

\begin{tabular}{|c|c|c|c|c|c|c|c|}
\hline Infection & Patient no. & Age & bla & Co-infection & Treatment & $\begin{array}{l}\text { Microbiological } \\
\text { outcome }\end{array}$ & Mortality \\
\hline \multirow[t]{17}{*}{ Pneumonia } & 1 & 54 & $\mathrm{KPC}$ & - & MER COL TIG & - & No \\
\hline & 2 & 67 & VIM-1 & - & MER COL TIG & - & No \\
\hline & 3 & 39 & VIM-1 & Bacteremia & MER COL TIG & - & No \\
\hline & 4 & 22 & VIM-1 & - & MER COL TIG & Failure & No \\
\hline & 5 & 52 & KPC & - & GEN COL TIG & - & No \\
\hline & 6 & & KPC & - & GEN COL TIG & - & No \\
\hline & 7 & 68 & $\mathrm{KPC}$ & - & GEN COL TIG & Failure & No \\
\hline & 8 & 19 & $\mathrm{KPC}$ & - & GEN COL TIG & Failure & No \\
\hline & 9 & 57 & $\mathrm{KPC}-2$ & UTI & GEN COL TIG & - & Yes \\
\hline & 10 & 63 & KPC-2 & - & GEN COL TIG & - & Yes \\
\hline & 11 & 52 & $\mathrm{KPC}-2$ & - & FOS COL TIG & Success & No \\
\hline & 12 & 28 & $\mathrm{KPC}-2$ & - & FOS COL TIG & Failure & No \\
\hline & 13 & 77 & $\mathrm{KPC}-2$ & Bacteremia, SSI & ERT DOR PMB & Success & No \\
\hline & 14 & 62 & $\mathrm{KPC}-2$ & - & ERT DOR COL PMB & Success & No \\
\hline & 15 & 62 & $\mathrm{KPC}-2$ & Bacteremia & DOR GEN COL TIG & Success & No \\
\hline & 16 & 67 & $\mathrm{KPC}-2$ & - & MERTIG RIF & Failure & Yes \\
\hline & 17 & 72 & $\mathrm{KPC}-2$ & - & MER FOS COL & Failure & Yes \\
\hline \multirow[t]{9}{*}{ Urinary tract } & 18 & 75 & OXA-48 & Bacteremia & MER ERT COL & Success & No \\
\hline & 19 & 63 & $\mathrm{KPC}-2$ & - & MER FOS COL & Failure & No \\
\hline & 20 & 68 & - & - & MER FOS COL DOX & Failure & No \\
\hline & 21 & 33 & - & - & MER FOS DOX & Success & No \\
\hline & 22 & 31 & - & Bacteremia & MER COL TIG & Failure & No \\
\hline & 23 & 62 & - & Bacteremia & FOS COL TIG & Failure & No \\
\hline & 24 & 54 & KPC & Bacteremia & DOR GEN FOS COL & Failure & No \\
\hline & 25 & 61 & KPC & Bacteremia & FOS TIG DOX & Failure & Yes \\
\hline & 26 & 70 & $\mathrm{KPC}-2$ & Bacteremia & AMK COL TIG & - & Yes \\
\hline \multirow[t]{11}{*}{ Primary or catheter-related bacteremia } & 27 & 61 & $\mathrm{KPC}-2$ & - & MERTIG CIP & Failure & No \\
\hline & 28 & 30 & $\mathrm{KPC}-2$ & - & MER FOS COL & Success & No \\
\hline & 29 & 42 & KPC-2 & - & MER GEN COL & Success & Yes \\
\hline & 30 & 69 & KPC-2 & - & MER COL CIP & Success & No \\
\hline & 31 & 73 & KPC-2 & - & GEN FOS TIG & Success & No \\
\hline & 32 & 46 & KPC-2 & - & AMK COL TIG TZP & Success & Yes \\
\hline & 33 & 64 & KPC-2 & - & MER COL TIG & Failure & No \\
\hline & 34 & 55 & $\mathrm{KPC}-2$ & - & MER COL TIG & Failure & Yes \\
\hline & 35 & 74 & VIM-1 & - & MER COL TIG & Failure & Yes \\
\hline & 36 & 63 & $\mathrm{KPC}-2$ & - & FOS COL TIG & Failure & Yes \\
\hline & 37 & 65 & $\mathrm{KPC}-2$ & - & COL CIPTZP & Failure & Yes \\
\hline \multirow[t]{6}{*}{ Intra-abdominal } & 38 & 44 & $\mathrm{KPC}-2$ & Bacteremia & GEN COL TIG & Success & No \\
\hline & 39 & 60 & KPC-2 & Bacteremia, SSI & GEN COL TIG & Success & Yes \\
\hline & 40 & 38 & $\mathrm{KPC}-2$ & - & GEN COL TIG & Success & No \\
\hline & 41 & 53 & $\mathrm{KPC}-2$ & Bacteremia & GEN COL FOS & Success & Yes \\
\hline & 42 & 57 & $\mathrm{KPC}$ & Bacteremia & GEN COL TIG & Failure & Yes \\
\hline & 43 & 75 & $\mathrm{KPC}$ & Bacteremia & FOS COL TIG TZP & Failure & Yes \\
\hline \multirow[t]{4}{*}{ Peritonitis } & 44 & 63 & $\mathrm{KPC}-2$ & - & AMKTIG CIP & Failure & No \\
\hline & 45 & 61 & $\mathrm{KPC}-2$ & - & AMK COL TIG & Success & No \\
\hline & 46 & 27 & $\mathrm{KPC}-2$ & Cholangitis & GEN COL TIG & - & Yes \\
\hline & 47 & 55 & $\mathrm{KPC}-2$ & - & AMK TZP SMT & Success & No \\
\hline
\end{tabular}


Table 3 continued

\begin{tabular}{|c|c|c|c|c|c|c|c|}
\hline Infection & Patient no. & Age & bla & Co-infection & Treatment & $\begin{array}{l}\text { Microbiological } \\
\text { outcome }\end{array}$ & Mortality \\
\hline \multirow[t]{2}{*}{ Surgical site } & 48 & 70 & KPC-2 & Bacteremia & AMK COL TIG & & Yes \\
\hline & 49 & 34 & $\mathrm{KPC}-2$ & - & ERT FOS COL & Success & No \\
\hline Ventriculitis & 50 & 43 & $\mathrm{KPC}-2$ & - & AMK GEN COL TIG & Success & No \\
\hline Meningitis & 51 & 18 & OXA-48 & - & FOS COL TZP & Success & No \\
\hline Lower respiratory & 52 & 39 & OXA-48 & - & AMK COL TIG & - & No \\
\hline Prosthetic joint & 53 & 58 & $\mathrm{KPC}$ & - & AMK COL TIG & Failure & Yes \\
\hline
\end{tabular}

bla beta-lactamase, SSI surgical site infection, UTI urinary tract infection, AMK amikacin, CIP ciprofloxacin, COL colistin, DOR doripenem, DOX doxycycline, ERT ertapenem, FOS fosfomycin, GEN gentamicin, MER meropenem, PMB polymyxin B, RIF rifampin, SMT sulfamethoxazole/trimethoprim, TIG tigecycline, TZP piperacillin/ tazobactam

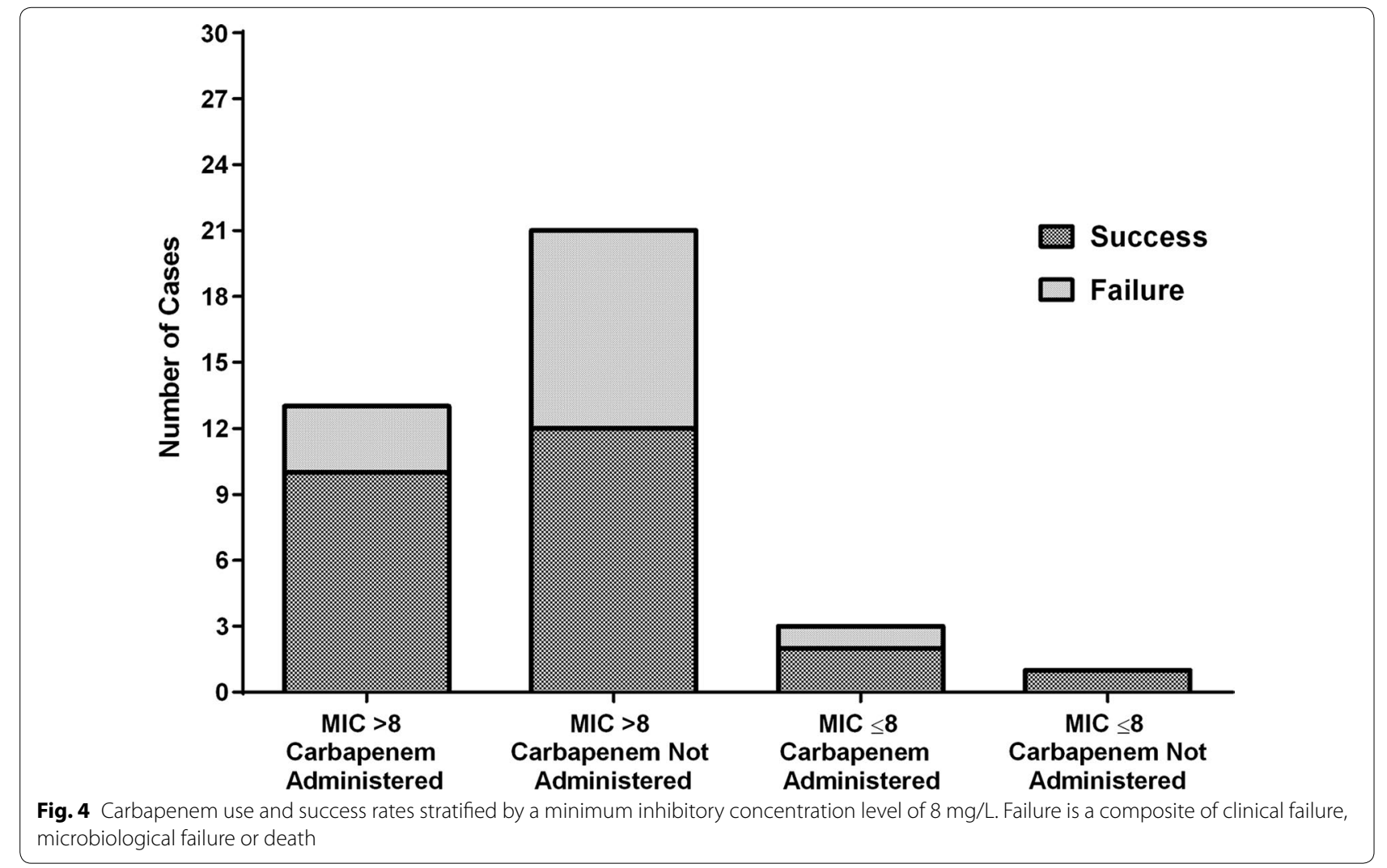

with a novel $\beta$-lactamase inhibitor approved by the US Food and Drug Administration (FDA) in February 2015 [60]. Ceftazidime-avibactam has shown potent in vitro activity against CRE isolates [61-63]. and there have also been reports that ceftazidime-avibactam is effective for CPKP infections after other combination regimens have failed $[19,64,65]$. Other $\beta$-lactam $/ \beta$-lactamase inhibitor combinations are also being investigated including ceftolozane-tazobactam and aztreonam-avibactam [12, 66]. Plazomicin, a novel aminoglycoside that has shown in vitro activity against $\mathrm{CRE}$, is currently undergoing a Phase 3 clinical trial (NCT01970371) as part of a combination therapy [67]. Another agent showing potential is eravacycline, a tetracycline derivative, which has shown in vitro efficacy against CRE as well as for complicated intra-abdominal infections and complicated urinary tract infections in clinical trials $[68,69]$.

There are several limitations of this review. First, there were a limited number of cases treated with triple combination therapy that were considered tier 1 . The purpose of this review was to explore the utilization of this treatment modality for CPKP infections; therefore we needed to be more stringent in our selection of cases. A number of articles did not provide sufficient detail to explore triple combination therapy and related outcomes, therefore we excluded them from the analysis. Second, the cases 
are heterogeneous in terms of their resistance mechanisms, MICs and antimicrobial therapies, which may limit the generalizability of our findings. The number of CPKP cases treated with triple antibiotic therapy are limited and it was our intention to include those that provided sufficient detail. Further research is necessary to explore specific antimicrobial regimens based on different levels of resistance for the treatment of CPKP. Finally, the timing of antibiotics differed among the studies with only a small number of patients receiving triple antibiotic therapy as empirical therapy. Accordingly, these differences may influence the clinical outcomes.

\section{Conclusions}

CPKP infections are a significant challenge worldwide, especially as infections caused by these organisms are associated with morbidity and high mortality. Few antimicrobials retain activity against CPKP, so polymyxinbased combinations have become an important treatment option for patients with these infections. Clinical data on the most appropriate antibiotic combination are sparse, and most of the evidence is based on observational studies. This review summarizes how triple combination therapy has been used against carbapenemase-producing $K$. pneumoniae infections. Triple combination therapy is being considered as a treatment option in the clinic and may be appropriate given the rise of polymyxin resistance. Further research is necessary to establish which treatment combination is superior and how to best utilize combination therapy for the treatment of CPKP infections.

\section{Authors' contributions}

DJ and GR conceptualized and designed the study. MCS, DH, FM, and AP collected and analyzed the data. DJ, GR, and MCS interpreted the analysis. DJ, GR and MCS drafted the manuscript. DJ and GR critically revised the manuscript. All authors read and approved the final manuscript.

\section{Acknowledgements}

Not applicable.

\section{Competing interests}

The authors declare that they have no competing interests.

\section{Availability of data and materials}

All data analyzed during this study are included in this published article.

Ethics approval and consent to participate

Not applicable.

Funding

None.

\section{Publisher's Note}

Springer Nature remains neutral with regard to jurisdictional claims in published maps and institutional affiliations.

Received: 13 June 2017 Accepted: 8 November 2017

Published online: 25 November 2017
References

1. Munoz-Price LS, Poirel L, Bonomo RA, et al. Clinical epidemiology of the global expansion of Klebsiella pneumoniae carbapenemases. Lancet Infect Dis. 2013;13(9):785-96.

2. Pitout JD, Nordmann P, Poirel L. Carbapenemase-producing Klebsiella pneumoniae, a key pathogen set for global nosocomial dominance. Antimicrob Agents Chemother. 2015;59(10):5873-84.

3. Falagas ME, Lourida P, Poulikakos P, Rafailidis PI, Tansarli GS. Antibiotic treatment of infections due to carbapenem-resistant Enterobacteriaceae: systematic evaluation of the available evidence. Antimicrob Agents Chemother. 2014;58(2):654-63.

4. Qureshi ZA, Paterson DL, Potoski BA, et al. Treatment outcome of bacteremia due to KPC-producing Klebsiella pneumoniae: superiority of combination antimicrobial regimens. Antimicrob Agents Chemother. 2012;56(4):2108-13.

5. Hoxha A, Karki T, Giambi C, et al. Attributable mortality of carbapenemresistant Klebsiella pneumoniae infections in a prospective matched cohort study in Italy, 2012-2013. J Hosp Infect. 2016;92(1):61-6.

6. Morrill HJ, Pogue JM, Kaye KS, LaPlante KL. Treatment options for carbapenem-resistant Enterobacteriaceae infections. Open Forum Infect Dis. 2015;2(2):ofv050.

7. Vardakas KZ, Tansarli GS, Rafailidis PI, Falagas ME. Carbapenems versus alternative antibiotics for the treatment of bacteraemia due to Enterobacteriaceae producing extended-spectrum beta-lactamases: a systematic review and meta-analysis. J Antimicrob Chemother. 2012:67(12):2793-803.

8. Canton R, Akova M, Carmeli Y, et al. Rapid evolution and spread of carbapenemases among Enterobacteriaceae in Europe. Clin Microbiol Infect. 2012;18(5):413-31.

9. Organization WH. Antimicrobial resistance: global report on surveillance. World Health Organization; 2014.

10. Nordmann P, Poirel L. The difficult-to-control spread of carbapenemase producers among Enterobacteriaceae worldwide. Clin Microbiol Infect. 2014;20(9):821-30.

11. Tzouvelekis LS, Markogiannakis A, Piperaki E, Souli M, Daikos GL. Treating infections caused by carbapenemase-producing Enterobacteriaceae. Clin Microbiol Infect. 2014;20(9):862-72.

12. Tumbarello M, Trecarichi EM, De Rosa FG, et al. Infections caused by KPCproducing Klebsiella pneumoniae: differences in therapy and mortality in a multicentre study. J Antimicrob Chemother. 2015;70(7):2133-43.

13. Petrosillo N, Giannella M, Lewis R, Viale P. Treatment of carbapenemresistant Klebsiella pneumoniae: the state of the art. Expert Rev Anti Infect Ther. 2013;11(2):159-77.

14. Rojas LJ, Salim M, Cober E, et al. Colistin resistance in carbapenem-resistant Klebsiella pneumoniae: laboratory detection and impact on mortality. Clin Infect Dis. 2017;64(6):711-8.

15. Giacobbe DR, Del Bono V, Trecarichi EM, et al. Risk factors for bloodstream infections due to colistin-resistant KPC-producing Klebsiella pneumoniae: results from a multicenter case-control-control study. Clin Microbiol Infect. 2015;21(12):1106-8.

16. Diep J, Jacobs DM, Sharma R, et al. Polymyxin B in combination with rifampin and meropenem against polymyxin B-resistant KPCproducing Klebsiella pneumoniae. Antimicrob Agents Chemother. 2017;61(2):e02121.

17. Gaibani P, Lombardo D, Lewis RE, et al. In vitro activity and post-antibiotic effects of colistin in combination with other antimicrobials against colistin-resistant KPC-producing Klebsiella pneumoniae bloodstream isolates. J Antimicrob Chemother. 2014;69(7):1856-65.

18. Ariza-Heredia EJ, Patel R, Blumberg EA, et al. Outcomes of transplantation using organs from a donor infected with Klebsiella pneumoniae carbapenemase (KPC)-producing K. pneumoniae. Transpl Infect Dis. 2012;14(3):229-36

19. Camargo JF, Simkins J, Beduschi T, et al. Successful treatment of carbapenemase-producing pandrug-resistant Klebsiella pneumoniae bacteremia. Antimicrob Agents Chemother. 2015;59(10):5903-8.

20. Chua NG, Zhou YP, Tan TT, et al. Polymyxin B with dual carbapenem combination therapy against carbapenemase-producing Klebsiella pneumoniae. J Infect. 2015;70(3):309-11.

21. de Sanctis J, Teixeira $L$, van Duin D, et al. Complex prosthetic joint infections due to carbapenemase-producing Klebsiella pneumoniae: a 
unique challenge in the era of untreatable infections. Int J Infect Dis, 2014;25:73-8

22. Giamarellou H, Galani L, Baziaka F, Karaiskos I. Effectiveness of a doublecarbapenem regimen for infections in humans due to carbapenemaseproducing pandrug-resistant Klebsiella pneumoniae. Antimicrob Agents Chemother. 2013;57(5):2388-90.

23. Lemmenmeier E, Kohler P, Bruderer T, Goldenberger D, Kleger GR, Schlegel M. First documented outbreak of KPC-2-producing Klebsiella pneumoniae in Switzerland: infection control measures and clinical management. Infection. 2014;42(3):529-34.

24. Marquez C, Ingold A, Echeverria N, et al. Emergence of KPC-producing Klebsiella pneumoniae in Uruguay: infection control and molecular characterization. New Microbes New Infect. 2014;2(3):58-63.

25. Mathers $\mathrm{AJ}$, Cox HL, Bonatti $\mathrm{H}$, et al. Fatal cross infection by carbapenem resistant Klebsiella in two liver transplant recipients. Transpl Infect Dis. 2009;11(3):257-65.

26. Nevrekar S, Cunningham KC, Greathouse KM, Panos NG. Dual intraventricular plus systemic antibiotic therapy for the treatment of Klebsiella pneumoniae carbapenemase-producing Klebsiella pneumoniae ventriculitis. Ann Pharmacother. 2014;48(2):274-8

27. Oliva A, Mascellino MT, Cipolla A, et al. Therapeutic strategy for pandrugresistant Klebsiella pneumoniae severe infections: short-course treatment with colistin increases the in vivo and in vitro activity of double carbapenem regimen. Int J Infect Dis. 2015;33:132-4.

28. Pontikis K, Karaiskos I, Bastani S, et al. Outcomes of critically ill intensive care unit patients treated with fosfomycin for infections due to pandrugresistant and extensively drug-resistant carbapenemase-producing Gram-negative bacteria. Int J Antimicrob Agents. 2014;43(1):52-9.

29. Viaggi B, Sbrana F, Malacarne P, Tascini C. Ventilator-associated pneumonia caused by colistin-resistant KPC-producing Klebsiella pneumoniae: a case report and literature review. Respir Investig. 2015;53(3):124-8.

30. Virgilio E, Castaldo P, Catta F, Tarantino G, Cavallini M. Abdominal surgical site infection due to Klebsiella pneumoniae carbapenemase-producing $K$. pneumoniae. Int Wound J. 2016;13(5):1075-6.

31. Balandin Moreno B, Fernandez Simon I, Pintado Garcia V, et al. Tigecycline therapy for infections due to carbapenemase-producing Klebsiella pneumoniae in critically ill patients. Scand J Infect Dis. 2014;46(3):175-80.

32. Cicora F, Mos F, Roberti J. Kidney transplant recipients infected with blaKPC-2-producing Klebsiella pneumoniae. Nefrologia. 2015;35(3):340-3.

33. Clancy CJ, Chen L, Shields RK, et al. Epidemiology and molecular characterization of bacteremia due to carbapenem-resistant Klebsiella pneumoniae in transplant recipients. Am J Transpl. 2013;13(10):2619-33.

34. Daikos GL, Tsaousi S, Tzouvelekis LS, et al. Carbapenemase-producing Klebsiella pneumoniae bloodstream infections: lowering mortality by antibiotic combination schemes and the role of carbapenems. Antimicrob Agents Chemother. 2014;58(4):2322-8.

35. Freire MP, Pierrotti LC, Filho HH, et al. Infection with Klebsiella pneumoniae carbapenemase (KPC)-producing Klebsiella pneumoniae in cancer patients. Eur J Clin Microbiol Infect Dis. 2015;34(2):277-86.

36. Kontopidou F, Giamarellou H, Katerelos P, et al. Infections caused by carbapenem-resistant Klebsiella pneumoniae among patients in intensive care units in Greece: a multi-centre study on clinical outcome and therapeutic options. Clin Microbiol Infect. 2014;20(2):0117-23.

37. Lubbert C, Becker-Rux D, Rodloff AC, et al. Colonization of liver transplant recipients with $\mathrm{KPC}$-producing Klebsiella pneumoniae is associated with high infection rates and excess mortality: a case-control analysis. Infection. 2014:42(2):309-16.

38. Maltezou HC, Giakkoupi P, Maragos A, et al. Outbreak of infections due to KPC-2-producing Klebsiella pneumoniae in a hospital in Crete (Greece). J Infect. 2009:58(3):213-9.

39. Morris D, Boyle F, Morris C, et al. Inter-hospital outbreak of Klebsiella pneumoniae producing KPC-2 carbapenemase in Ireland. J Antimicrob Chemother. 2012;67(10):2367-72.

40. Mouloudi E, Massa E, Piperidou M, et al. Tigecycline for treatment of carbapenem-resistant Klebsiella pneumoniae infections after liver transplantation in the intensive care unit: a 3-year study. Transpl Proc. 2014;46(9):3219-21.

41. Navarro-San Francisco C, Mora-Rillo M, Romero-Gomez MP, et al. Bacteraemia due to OXA-48-carbapenemase-producing Enterobacteriaceae: a major clinical challenge. Clin Microbiol Infect. 2013;19(2):E72-9.
42. Sbrana F, Malacarne P, Viaggi B, et al. Carbapenem-sparing antibiotic regimens for infections caused by Klebsiella pneumoniae carbapenemase-producing K. pneumoniae in intensive care unit. Clin Infect Dis. 2013;56(5):697-700.

43. Souli M, Galani I, Antoniadou A, et al. An outbreak of infection due to beta-lactamase Klebsiella pneumoniae carbapenemase 2-producing $\mathrm{K}$. pneumoniae in a Greek University Hospital: molecular characterization, epidemiology, and outcomes. Clin Infect Dis. 2010;50(3):364-73.

44. van Duin D, Cober E, Richter SS, et al. Impact of therapy and strain type on outcomes in urinary tract infections caused by carbapenem-resistant Klebsiella pneumoniae. J Antimicrob Chemother. 2015;70(4):1203-11.

45. Papadimitriou-Olivgeris $M$, Marangos $M$, Christofidou M, et al. Risk factors for infection and predictors of mortality among patients with KPCproducing Klebsiella pneumoniae bloodstream infections in the intensive care unit. Scand J Infect Dis. 2014;46(9):642-8.

46. Pouch SM, Kubin CJ, Satlin MJ, et al. Epidemiology and outcomes of carbapenem-resistant Klebsiella pneumoniae bacteriuria in kidney transplant recipients. Transpl Infect Dis. 2015;17(6):800-9.

47. Sanchez-Romero I, Asensio A, Oteo J, et al. Nosocomial outbreak of VIM1-producing Klebsiella pneumoniae isolates of multilocus sequence type 15: molecular basis, clinical risk factors, and outcome. Antimicrob Agents Chemother. 2012;56(1):420-7.

48. Zarkotou O, Pournaras S, Tselioti P, et al. Predictors of mortality in patients with bloodstream infections caused by KPC-producing Klebsiella pneumoniae and impact of appropriate antimicrobial treatment. Clin Microbiol Infect. 2011;17(12):1798-803.

49. Urban C, Bradford PA, Tuckman M, et al. Carbapenem-resistant Escherichia coli harboring Klebsiella pneumoniae carbapenemase betalactamases associated with long-term care facilities. Clin Infect Dis. 2008;46(11):e127-30.

50. McGuinn M, Hershow RC, Janda WM. Escherichia coli and Klebsiella pneumoniae carbapenemase in long-term care facility, Illinois, USA. Emerg Infect Dis. 2009;15(6):988-9.

51. Woodford N, Wareham DW, Guerra B, Teale C. Carbapenemase-producing Enterobacteriaceae and non-Enterobacteriaceae from animals and the environment: an emerging public health risk of our own making? J Antimicrob Chemother. 2014;69(2):287-91.

52. Gijon D, Curiao T, Baquero F, Coque TM, Canton R. Fecal carriage of carbapenemase-producing Enterobacteriaceae: a hidden reservoir in hospitalized and non hospitalized patients. J Clin Microbiol. 2012;50(5):1558-63.

53. Capone A, Giannella M, Fortini D, et al. High rate of colistin resistance among patients with carbapenem-resistant Klebsiella pneumoniae infection accounts for an excess of mortality. Clin Microbiol Infect. 2013;19(1):E23-30.

54. Villa L, Feudi C, Fortini D, Garcia-Fernandez A, Carattoli A. Genomics of KPC-producing Klebsiella pneumoniae sequence type 512 clone highlights the role of RamR and ribosomal S10 protein mutations in conferring tigecycline resistance. Antimicrob Agents Chemother. 2014;58(3):1707-12.

55. Hauck C, Cober E, Richter SS, et al. Spectrum of excess mortality due to carbapenem-resistant Klebsiella pneumoniae infections. Clin Microbiol Infect. 2016;22(6):513-9.

56. Akova M, Daikos GL, Tzouvelekis L, Carmeli Y. Interventional strategies and current clinical experience with carbapenemase-producing Gramnegative bacteria. Clin Microbiol Infect. 2012;18(5):439-48.

57. Abboud CS, Monteiro J, Stryjewski ME, et al. Post-surgical mediastinitis due to carbapenem-resistant Enterobacteriaceae: clinical, epidemiological and survival characteristics. Int J Antimicrob Agents. 2016;47(5):386-90

58. Velkov T, Thompson PE, Nation RL, Li J. Structure-activity relationships of polymyxin antibiotics. J Med Chem. 2010;53(5):1898-916.

59. Lee J, Patel G, Huprikar S, Calfee DP, Jenkins SG. Decreased susceptibility to polymyxin B during treatment for carbapenem-resistant Klebsiella pneumoniae infection. J Clin Microbiol. 2009;47(5):1611-2.

60. Actavis. Actavis receives U.S. FDA approval for AVYCAZ ${ }^{\mathrm{TM}}$ (CEFTAZIDIMEAVIBACTAM). 2015. http://www.prnewswire.com/news-releases/actavisreceives-us-fda-approval-for-avycaz-ceftazidime-avibactam-300041592. html. Accessed 24 June 2016.

61. Sader HS, Castanheira M, Flamm RK, Mendes RE, Farrell DJ, Jones RN. Ceftazidime/avibactam tested against Gram-negative bacteria from 
intensive care unit (ICU) and non-ICU patients, including those with ventilator-associated pneumonia. Int J Antimicrob Agents. 2015;46(1):53-9.

62. Castanheira M, Mills JC, Costello SE, Jones RN, Sader HS. Ceftazidime-avibactam activity tested against Enterobacteriaceae isolates from U.S. hospitals (2011 to 2013) and characterization of beta-lactamase-producing strains. Antimicrob Agents Chemother. 2015;59(6):3509-17.

63. Vasoo S, Cunningham SA, Cole NC, et al. In Vitro Activities of ceftazidimeavibactam, aztreonam-avibactam, and a panel of older and contemporary antimicrobial agents against carbapenemase-producing gramnegative Bacilli. Antimicrob Agents Chemother. 2015;59(12):7842-6.

64. Caravaca-Fontan F, Jimenez-Alvaro S, Marcen-Letosa R, FernandezRodriguez A, Rodriguez-Navarro CQ. Ceftazidime-avibactam in urinary tract infections due to carbapenemase-producing Klebsiella in kidney transplantation. Nefrologia. 2015;35(4):412-3.
65. Jacobs DM, DiTursi S, Ruh C, et al. Combination treatment with extendedinfusion ceftazidime/avibactam for a KPC-3-producing Klebsiella pneumoniae bacteraemia in a kidney and pancreas transplant patient. Int J Antimicrob Agents. 2016;48(2):225-7.

66. Papp-Wallace KM, Bonomo RA. New beta-lactamase inhibitors in the clinic. Infect Dis Clin North Am. 2016;30(2):441-64.

67. Galani I, Souli M, Daikos GL, et al. Activity of plazomicin (ACHN-490) against MDR clinical isolates of Klebsiella pneumoniae, Escherichia coli, and Enterobacter spp. from Athens, Greece. J Chemother. 2012;24(4):191-4.

68. Livermore DM, Mushtaq S, Warner M, Woodford N. In vitro activity of eravacycline against carbapenem-resistant Enterobacteriaceae and Acinetobacter baumannii. Antimicrob Agents Chemother. 2016;60(6):3840-4.

69. Zhanel GG, Cheung D, Adam H, et al. Review of eravacycline, a novel fluorocycline antibacterial agent. Drugs. 2016;76(5):567-88.

\section{Submit your next manuscript to BioMed Central and we will help you at every step:}

- We accept pre-submission inquiries

- Our selector tool helps you to find the most relevant journal

- We provide round the clock customer support

- Convenient online submission

- Thorough peer review

- Inclusion in PubMed and all major indexing services

- Maximum visibility for your research

Submit your manuscript at www.biomedcentral.com/submit 